\title{
Effect of vibration on pain during Injection of local anesthesia: A split-mouth randomized clinical trial
}

\author{
Gholam Hossain Ramezani', Mohammad Tajjedin², Nasser Valaee ${ }^{3}$ and Hooman Ebrahimi** \\ ${ }^{1}$ Department of Pediatric Dentistry, Dental Branch, Islamic Azad University, Tehran, Iran \\ ${ }^{2}$ Specialist in Pediatric Dentistry \\ ${ }^{3}$ Epidemiologist, Dental Branch, Islamic Azad University, Tehran, Iran \\ ${ }^{4}$ Department of Oral Medicine, Dental Branch, Islamic Azad University, Tehran, Iran
}

\begin{abstract}
Vibration can reduce pain. This study was conducted to use this effect while injecting dental anesthesia. This splitmouth randomized clinical trial was performed on 40 injection sites from 20 patients. In the experimental sides, the turned-on device would be positioned in contact with the injection area for 5 seconds; then the anesthesia would be administered, while the device was in place; finally, the device would remain in place for 5 seconds after removing the needle. In the control sides, the device would be placed on the mucosa in a similar fashion but turned off. Immediately after removing the tip of Dental Vibe, patients were asked to rate their pain using Wong Baker method. Pain scores were compared statistically. Average pain levels in the experimental and control sides were $1.95 \pm 1.57(95 \%$ CI: 1.22 to 2.68 ) and $0.65 \pm 0.81$ (95\% CI: 0.27 to 1.03 ), respectively. Their difference was significant according to Wilcoxon test $(\mathrm{P}<0.001)$. Age $(\mathrm{P}=0.670)$, injection type $(\mathrm{P}=0.175)$, and sex $(\mathrm{P}=0.160)$ did not affect the response to the Dental Vibe significantly, according to chi-square test. Dental Vibe is a useful and effective device in reducing pain while injecting local anesthesia.
\end{abstract}

\section{INTRODUCTION}

Most common anxiety provoking and fearful experience for children in dental operatory is administration of local anesthesia. Pain management when injecting local anesthesia (LA) is one of the most critical stages in performing dental treatments. Since painful dental treat-

\section{ARTICLE INFORMATION:}

*Corresponding Author:

Received $10^{\text {th }}$ Oct, 2017

Accepted after revision $29^{\text {th }}$ Dec, 2017

BBRC Print ISSN: 0974-6455

Online ISSN: 2321-4007 CODEN: USA BBRCBA

. Thomson Reuters ISI ESC and Crossref Indexed Journal

NAAS Journal Score 2017: 4.31 Cosmos IF: 4.006

- A Society of Science and Nature Publication, 2017. All rights reserved.

Online Contents Available at: http//www.bbrc.in/

DOI: $10.21786 / \mathrm{bbrc} / 10.4 / 18$ ments typically begin with LA, pain control at this step is essential. Pain is a deterrent of dental treatment and many patients avoid or cancel their treatments because of this factor because of being afraid of pain, which is usually caused by previous painful experiences during dental procedures. Therefore, reducing dental pain is important and researchers have been seeking methods 
to reduce dental pain, including pain of injecting local anesthesia Milgrom et al., 1997, Yenisey 2009 Bonjar 2011, Ungor et al 2014, Rakshan and Rakshan 2015, Agarwal et al 2017).

Mechanisms for pain due to local anesthesia are mainly quick expansion of the tissues and their tension, followed by mechanical trauma by the needle puncture to the region of the injection. Various methods have been proposed to reduce or prevent pain while administering local anesthesia, including the application of topical anesthetics, suggestion, slow injection, transcutaneous electrical nerve stimulation (TENS) computer-assisted local anesthesia (such as Wand), and vibration (Hersh et al. 1996 Peretz et al., 2004 and Primosch 2003).

According to the "gate control" theory, providing non-noxious stimuli might interrupt nociceptive signals reduce the perceived pain . Thus, it is hypothesized that stimulating larger-diameter A-beta fibers with vibration and pressure might reduce pain sensation (Saijo et al., 2005 Nanitsos et al., 2009 and Rakshan and Rakshan 2015).

The Dental Vibe device (BING Innovations, Florida, USA) is a new portable system that transmits pressurerotational pulses to the injection area, without any need to changing the routine protocols of injection. We hypothesized that vibration concurrent with injection might decrease perceived pain in children.

\section{MATERIALS AND METHODS}

This split-mouth randomized clinical trial was performed on 40 injection sites from 20 patients (13 boys and 7 girls, with an average age of 5.7 years old) referred to the Department of Pediatric Dentistry at Islamic Azad University during 2014-2015. The protocol ethics were approved by the Ethical Committee of the university, and informed consents were taken from patients' parents. Inclusion criteria were being systemically healthy, being aged between 5 and 7 years old, a cooperation level of 3 or 4 (according to Frankel scale) determined by a pediatric dentist, ability to determine pain levels according to Wong Baker scale (8thesis), and clinical need for bilateral local anesthetic injection in the mandible or maxilla.

\section{Randomization, Local anesthesia administration, and Pain evaluation}

Randomization was performed by picking out a card from a box, for each patient. Patients with "vibration on" cards would receive the injection together with the DentalVibe application while the device was on. They would receive the second injection on the contralateral side, with the vibration off. The patients with "vibra- tion off" cards would first receive the placebo (the device touch without vibration) first, and the treatment in the second session.

In the experimental sides, the turned-on device would be positioned in contact with the injection area for $5 \mathrm{sec}-$ onds; then the anesthesia would be administered, while the device was in place; finally, the device would remain in place for 5 seconds after removing the needle. In the control sides, the device would be placed on the mucosa in a similar fashion but turned off. The inferior alveolar nerve (IAN) blocks were administered using a carpul of 2\% lidocaine and 1:80000 epinephrine (Darupakhsh, Tehran, Iran). The infiltration blocks were administered for the maxilla, using a carpul of the same anesthetic solution. Of the 40 injections, 22 were IAN blocks while 18 were maxillary infiltration injections.

Immediately after removing the tip of Dental Vibe, patients were asked to rate their pain using Wong Baker method. All injections were performed by the same person (a resident of pediatric dentistry).After data collection, the treatment would be started in its routine fashion; the patient would receive as many carpules as needed/wanted after the data collection.

After summarizing the descriptive statistics and confidence intervals (CI), Wilcoxon test and chi-square tests used to compare control and treatment groups.

\section{RESULTS AND DISCUSSION}

Of the 20 participants, 65\% reported lower pain levels when using the device; $25 \%$ rated the pain on both sides similarly; 10\% reported greater pain levels on the control sides. Average pain levels in the experimental and control sides were $1.95 \pm 1.57$ (95\% CI: 1.22 to 2.68 ) and $0.65 \pm 0.81$ (95\% CI: 0.27 to 1.03 ), respectively. Their difference was significant according to Wilcoxon test (P $<0.001)$.

Age $(\mathrm{P}=0.670)$, injection type $(\mathrm{P}=0.175)$, and sex $(\mathrm{P}$ $=0.160)$ did not affect the response to the Dental Vibe significantly, according to chi-square test (Table 1).

Table 1. Results pertaining to experimental sides in which the device was turned on $(n=20$, control sides are not used or shown).

\begin{tabular}{|l|l|l|l|l|l|}
\hline Factor & & Painless & Painful & RR & AR \\
\hline Gender & Boy & 9 & 4 & 3.6 & 37 \\
\hline & Girl & 2 & 5 & & \\
\hline Age & 5 yr old & 5 & 6 & 2.4 & 21 \\
\hline & $>5$ yr old & 3 & 6 & & \\
\hline Injection & IAN block & 8 & 3 & 3.5 & 30 \\
\hline & Infiltration & 3 & 6 & & \\
\hline
\end{tabular}


Dental anxiety and fear are the most frequent reasons preventing patients from dental visits, and are usually a byproduct of local anesthesia injections. Hence, pain and anxiety control during local anesthetic injections is of significant clinical importance, (Ungor et al., 2014, Bonjar 2011 Berggren and Meynert 1984). Topical anesthetics numb the injection surface and provide pain relief on needle insertion, although there are other factors which should be controlled (such as the clinician's expertise and amount, type, and dose of the injected medicine) for a complete pain control. Although this method reduces the pain during needle insertion, total elimination of injection pain relies on causes like the amount, type, and injection speed of anesthesia plus the experience of clinician. In addition, local anesthetics have narrow potential to enter deep into tissue. These might reduce the discomfort during insertion of needle through the surface however, they are not as effective when needle passes through deeper layers, (Singh and Roberts 1994, Meechan et al., 1998 Ungor et al 2014).

Hence, methods such as Wand and TENS are introduced to solve this. TENS triggers large-diameter nerves that are more sensitive to electrical stimuli than do smaller-diameter nerves. The result is closure of central gating mechanism to signals coming through nerves with smaller diameters. The same mechanism of gate control works for the vibration, which has impulses that are transmitted very fast (75 meters per second) through myelinated, thick, A-beta nerves. On the other hand, sense of pain travels at a 2 meters per second speed through unmyelinated and thin C fibers, ( Ungor et al., 2014, Nanitsos et al., 2009, Hall and Guyton 2015).

Simultaneous transmission of vibration signals through thick A-beta fibers versus pain signals through C fibers will make the sensory area of the brain release inhibitory neurotransmitters these and inhibit the activation of projection neurons within dorsal horn of spinal cord, leading to gate closure over pain stimuli. This is the reason vibration is used to reduce pain during many painful medical and dental procedures (Reed 2001, Ungor et al., 2014). Another factor that contributes to perception of pain is psychological status of person, particularly his or her fear or anxiety of pain. Dental fear can prolong and intensify the pain (Peretz et al 2004).

Our results are similar to those of Ungor et al. (2014) and Nanitsos et al. (2009) although in the latter study, source of vibration was extra-oral, which might decrease the efficacy of expected gate control mechanism because of pain and vibrated sites being distant. Another study done by Saijo et al. (1995) examined injection pain together with vibration of the site using VibraJect. They could not find significant differences between control and treatment groups. Difference could be due to different devices and methods.

\section{CONCLUSION}

This study has found DentalVibe as a useful and effective device in reducing pain while injecting local anesthesia.

\section{REFERENCES}

Agarwal N, Dhawan J, Kumar D, Anand A, Tangri K. 2017 Effectiveness of two aenesthetic agents used along with audio visual aids in pediatric dental patients. Journal of clinical and diagnostic research. 11(1):80

Berggren U, Meynert G. 1984 Dental fear and avoidance: causes, symptoms, and consequences. The Journal of the American Dental Association. 1984 08;109(2):247-51.

Bjerring P, Arendt-Nielsen L. 1990 Depth and duration of skin analgesia to needle insertion after topical application of EMLA cream. British Journal of Anaesthesia. 64(2):173-7.

Haas DA. 2002 An update on local anesthetics in dentistry. Journal-Canadian Dental Association. 68(9):546-52.

Hall JE. Guyton JM 2015 A textbook of medical physiology: Elsevier Health Sciences; 2015.

Hersh EV, Houpt MI, Cooper SA, Feldman RS, Wolff MS, Levin LM. 1996 Analgesic Efficacy And Safety Of An Intraoral Lidocaine Patch. The Journal of the American Dental Association. /11;127(11):1626-34.

Hutchins HS, Jr., Young FA, Lackland DT, Fishburne CP. 1997 The effectiveness of topical anesthesia and vibration in alleviating the pain of oral injections. Anesth Prog. Summer;44(3):87-9.

Johnson J, Primosch RE.2003 Influence of site preparation methods on the pain reported during palatal infiltration using the Wand Local Anesthetic System. Am J Dent. 16(3):165-9.

Malamed SF. 2006 Local anesthetics: dentistry's most important drugs, clinical update CDA J. 2006;34(12):971-6.

Meechan JG, Gowans AJ, Welbury RR. 1998 The use of patientcontrolled transcutaneous electronic nerve stimulation (TENS) to decrease the discomfort of regional anaesthesia in dentistry: a randomised controlled clinical trial. Journal of Dentistry. 07;26(5-6):417-20.

Melzack R, Wall PD. 1965 Pain Mechanisms: A New Theory. Science. 1965 11/19;150(3699):971-8.

Milgrom P, Coldwell SE, Getz T, Weinstein P, Ramsay DS. 1997 Four Dimensions Of Fear Of Dental Injections. The Journal of the American Dental Association 06;128(6):756-62.

Nanitsos E, Vartuli R, Forte A, Dennison PJ, Peck CC. 2009 The effect of vibration on pain during local anaesthesia injections. Australian Dental Journal. 06;54(2):94-100.

Peretz B, Nazarian Y, Bimstein E. 2004 Dental anxiety in a students' paediatric dental clinic: children, parents and students. International Journal of Paediatric Dentistry. /05;14(3):192-8.

Quarnstrom F, Libed EN. 1994 Electronic anesthesia versus topical anesthesia for the control of injection pain. Quintessence Int;25(10):713-6. 
Rakhshan H, Rakhshan V. 2015 Pain and discomfort perceived during the initial stage of active fixed orthodontic treatment. The Saudi dental journal. 27(2):81-7.

Reed ML. 2001 Surgical Pearl: Mechanoanesthesia to reduce the pain of local injections. Journal of the American Academy of Dermatology. 2001 /04;44(4):671-2.

Saijo M, Ito E, Ichinohe T, Kaneko Y. 2005 Lack of Pain Reduction by a Vibrating Local Anesthetic Attachment: A Pilot Study. Anesthesia Progress. 2005 06;52(2):62-4.

Shahidi Bonjar AH. 2011 Syringe micro vibrator (SMV) a new device being introduced in dentistry to alleviate pain and anxiety of intraoral injections, and a comparative study with a similar device. Annals of Surgical Innovation and Research;5(1):1.

Sharma P, Czyz CN, Wulc AE. 2011 Investigating the Efficacy of Vibration Anesthesia to Reduce Pain From Cos- metic Botulinum Toxin Injections. Aesthetic Surgery Journal./10/14;31(8):966-71.

Singh P, Roberts M.1994 Skin permeability and local tissue concentrations of nonsteroidal anti-inflammatory drugs after topical application. Journal of Pharmacology and Experimental Therapeutics. 268(1):144-51.

Ungor C, Tosun E, Dayisoylu EH, Taskesen F, Senel FC. 2014 The effects of vibration on pain and anxiety during local anesthesia administration. JSM Dent. 2(1):1022.

Walton RE, Torabinejad M. Principles and practice of endodontics. 1996.

Yenisey M. 2009 Comparison of the pain levels of computer-controlled and conventional anesthesia techniques in prosthodontic treatment. Journal of Applied Oral Science. 2009/10;17(5). 